Rev. Bras. Cir. Cardiovasc.,

2(3): $180-188,1987$.

\title{
Retroca valvular
}

Pablo M. A. POMERANTZEFF*, Mário César S. de ABREU*, Marisa AMATO*, Miguel MORETTI*, José Otávio C. AULER JÚNIOR ${ }^{*}$, Max GRINBERG, ${ }^{*}$, Flávio TARASOUTCHI*, Alfredo MANSUR ${ }^{*}$, Altamiro Ribeiro DIAS*, Delmont BITTENCOURT*, Noedir A. G. STOLF*, Geraldo VERGINELLI*, Adib D. JATENE*

RBCCV 44205-39

POMERANTZEFF, P. M. A.; ABREU, M. C. S.; AMATO, M.:MORETTI, M.; AULER JÚNIOR, J. O. C.: GRINBERG, M.; TARASOUTCHI, F.; MANSUR, A.; DIAS, A. R.; BITTENCOURT, D.; STOLF, N. A. G.; VERGINELLI, G.; JATENE, A. D. - Retroca valvular. Rev. Bras. Cir. Cardiovasc., 2(3) 180-188, 1987.

RESUMO: A reoperação de próteses valvulares tem sido realizada, com freqüência cada vez maior, nos vários Serviços de cirurgia cardiaca. Os detalhes do tratamento, a indicação e a técnica operatória melhoraram os resultados. No periodo de janeiro de 1984 a junho de 1986 , no Instituto do Coração, foram submetidos a retroca valvular 145 pacientes, num total de 157 próteses, e 4 trocas da bola de válvula de Starr-Edwards. Em posição mitral, 6 pacientes foram submetidos a terceira troca valvular, sem óbito imediato. A insuficiência valvular e a calcificação do tecido biológico de dura-máter foram as principais causas de indicação da reoperação. Quarenta e um pacientes apresentavam roturas e 19 pacientes, calcificação de bioprótese em posição mitral; em posição aórtica, 32 pacientes tinham rotura e 12, calcificação de bioprótese. Quanto à prótese implantada, foram utilizadas principalmente as biopróteses, sendo 63 porcinas e 35 de pericárdio bovino. A mortalidade imediata global foi de $8.3 \%$ (12 pacientes), sendo a principal causa de óbito o baixo débito cardiaco. As principais complicaçóes imediats foram: baixo débito cardiaco, arritmias e sangramento. Noventa por cento dos pacientes encontravam-se em classe funcional (NYHA) III e IV no pré-operatório, evoluindo para as classes funcionais I e II em $89 \%$ das trocas aórticas de $82 \%$ das trocas mitrais. A curva atuarial de sobrevida,em 5 semestres, foi, para a posição mitral, de $85,7 \%$ e, para a aórtica, de $91,3 \%$. Os autores concluem que os cuidados de técnica, a proteção miocárdica e o tipo de prótese utilizada foram os responsáveis pelos resultados bastante satisfatórios.

DESCRITORES: próteses valvulares cardiacas, cirurgia, retroca.

\section{INTRODUÇÃO}

Como já foi demonstrado por vários autores ${ }^{1.14,15}$, a substituição valvar modificou, de muito, a história natural da doença valvar grave, aumentando a sobrevida e melhorando a qualidade de vida dos pacientes.

A partir das primeiras próteses desenvolvidas por EDWARDS \& STARR, na década de 1950 (STARR ${ }^{17}$ ), novos modelos e várias modificações têm sido introduzidas, na tentativa de aumentar a sua vida média. Muitas complicações têm sido descritas, após a substituição valvar, destacando-se, nas biopróteses, as relacionadas ao tecido, tais como a rotura e a calcificação, e, nas próteses mecânicas, o tromboembolismo ou os problemas relacionados ao oclusor. A calcificação ocorre, predominantemente, em pacientes mais jovens ${ }^{21}$.

Em novvo Serviço, foi utilizada, até 1982, a bioprótese de dura-máter, introduzida por PUIG et alii ${ }^{14}$, em 1970. Seguiu-se a este período o uso das biopróteses de pericárdio bovino e porcinas tratadas com glutaraldeído.

Dado o grande número de próteses implantadas, aumenta, também, a cada dia, o número de pacientes submetidos a retroca valvular.

Trabalho realizado no Instituto do Coração do Hospital das Clínicas da Faculdade de Medicina da Universidade de Sảo Paulo, São Paulo, SP. Brasil.

Apresentado ao 14: Congresso Nacional de Cirurgia Cardiaca. Salvador, BA, 27 e 28 de março, 1987, pelo Dr. Mário César S. de Abreu

- Do Instituto do Coração do Hospital das Clinicas da Faculdade de Medicina da Universidade de São Paulo.

Endereço para separatas: Pablo Pomerantzeff. Av.Dr. Eneas Carvalho de Aguiar, 44. Divisào Cirúrgica. 05403 São Paulo, SP. Brasił. 
Neste trabalho, é estudado um grupo de pacientes submetido a retroca valvular, no periodo de janeiro de 1984 a junho de 1986, analizando a causa das reoperações, as complicações e a evolução dos pacientes.

\section{CASUÍSTICA E MÉTODOS}

No periodo de janeiro de 1984 a junho de 1986, foram submetidos a retroca valvular 145 pacientes, com idade média de 36 anos, mínima de 10 e máxima de 76 anos. Nāo houve grande variaçāo entre a idade média dos pacientes submetidos a retroca valvular isolada ou combinada (Gráfico 1). Foram $76(52,4 \%)$ pacientes do sexo masculino e $69(47,6 \%)$ do sexo femino.

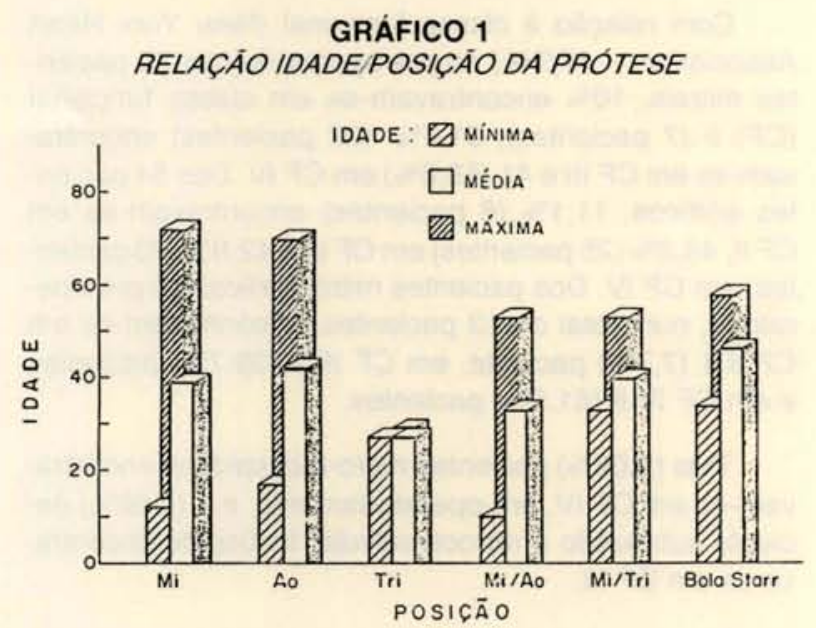

Deste total, foram submetidos a retroca valvular mitral isolada $70(48,2 \%)$ pacientes, sendo $20(28,5 \%)$ do sexo masculino e $50(71,4 \%)$ do sexo feminino. O total de retroca valvular aórtica isolada foi de 54 (37,9\%) pacientes, sendo $40(74,1 \%)$ do sexo masculino e 14 $(25,9 \%)$ do sexo feminino. Em posição tricúspide apenas $1(0,6 \%)$ paciente do sexo masculino foi submetido a retroca isolada.

Em $16(11,03 \%)$ pacientes, foram substituídas 2 válvulas, sendo $13(8,9 \%)$ pacientes do sexo masculino. Os outros $3(2,06 \%)$ pacientes eram mitro-tricuspídeos, $1(33,3 \%)$ paciente do sexo masculino e $2(66,6 \%)$ pcientes do sexo feminino (Gráfico 2).

Foram utilizadas um total de 161 próteses, assim distribuídas: $63(39,1 \%)$ biopróteses de porco tipo Biocor, sendo $34(53,9 \%)$ em posiçãomitral, $28(44,4 \%)$ em posição aórtica, 1 (1,58\%) em posição tricuspídea; 7 (4,3\%) biopróteses de porco tipo Labcor, sendo 4 (57,1\%) em posição mitral, e $3(42,8 \%)$ em posição aórtica; 57 $(35,4 \%)$ biopróteses de pericárdio bovino tipo EBM-Incor de baixo perfil, sendo $36(63,1 \%)$ em posição mitral, $19(33,3 \%)$ em posição aórtica, $2(3,5 \%)$ na posiçāo tricúspide; 4 (2,48\%) biopróteses de pericárdio bovino de alto perfil, sendo $1(25 \%)$ em posição mitral e $3(75 \%)$ em posição aórtica; $19(11,8 \%)$ biopróteses de porto tipo Barone, sendo $10(52,6 \%)$ em posição mitral, 8 $(42,1 \%)$ em posição aórtica e $1(5,2 \%)$ prótese em posição tricúspide: $7(43,4 \%)$ próteses mecânicas, sendo 4 de disco basculante, 1 de tipo Omnicarbon em posição mitral, 3 de tipo Sorin em posição aórtica, e 3 próteses de bola de tipo Starr-Edwards em posiçāo aórtica. Em $4(2,5 \%)$ pacientes portadores de próteses de Starr-Edwards em posição aórtica, foi feita apenas a troca da bola.

GRÁFICO 2

RELAÇÃO SEXO NÚMERO DE PRÓTESES

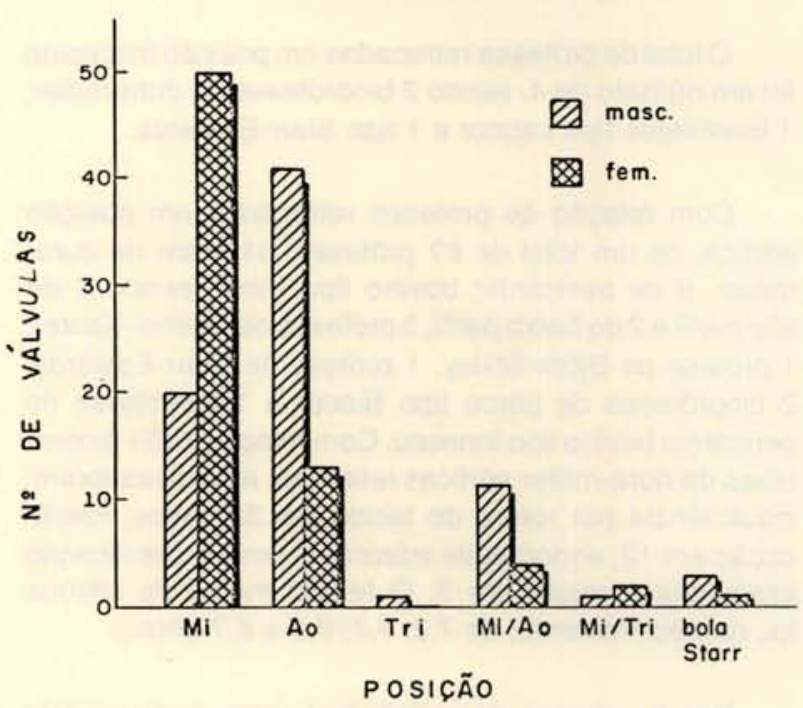

Do total de próteses retrocadas em posição mitral, $66(75 \%)$ eram e dura-máter, com um tempo médio de implante de 7,3 anos, mínimo de 3 e máximo de 14 . Neste grupo, por insuficiência devido à rotura do tecido, foram operados $41(62,12 \%)$ casos, por calcificação 20 $(22,7 \%), 1$ destes associado a rotura do tecido. Por endocardite infecciosa foram realizadas $5(5,7 \%)$ retrocas. Nos casos de insuficiência devido a rotura do tecido, o tempo médio de retroca foi de 7,1 anos, nos casos de calcificação foi de 7,0 anos e nos de endocardite, 7,7 anos. Ainda em posição mitral, $13(14,7 \%)$ próteses de pericárdio bovino foram retrocadas, sendo 10 biopróteses tipo Incor de alto perfil, 1 de baixo perfil, 1 tipo Macchi e 1 tipo lonescu. Das próteses de pericárdio bovino modelo Incor de alto perfil, 3 foram retrocadas devido a endocardite infecciosa, 3 devido a rotura, 2 por escape paravalvular e 2 por calcificação. O tempo médio de retroca foi, respectivamente, de $6 ; 1 ; 2,5$ e 3,5 anos. Uma bioprótese de pericárdio bovino modelo Incor de baixo perfil foi retrocada, após 1 ano, por defeito de confecção, 1 bioprótese de pericárdio bovino tipo 
POMERANTZEFF, P. M. A.; ABREU. M. C. S.; AMATO, M.: MORETTI, M.; AULER JÚNIOR, J. O. C.: GRINBERG, M.; TARASOUTCHI. F.; MANSUR, A.; DIAS, A. R.; BITTENCOURT, D.; STOLF, N. A. G.; VERGINELLI, G.; JATENE, A. D. - Retroca valvular. Rev. Bras. Cir. Cardiovasc., 2(3): 180-188, 1987.

Macchi foi retrocada, após 8 anos, por rotura, 1 bioprótese de pericárdio bovino tipo lonescu, por endocardite infecciosa. Foram em número de 6 as próteses biológicas de porco retrocadas em posição mitral: 4 biopróteses Biocor, uma do tipo Barone e uma do tipo CarpentierEdwards. Duas biopróteses Biocor foram substituidas, após 4 anos, devido a calcificação, 1 após 2 anos, por endocardite infecciosa, e outra por escape paravalvular, após 1 ano. A bioprótese do tipo Barone foi retrocada após 3 anos, por insuficiência, e a bioprótese de Carpentier-Edwards, por calcificação, não se identificando o tempo de implante. Duas próteses de Starr-Edwards em posição mitral necessitaram de troca, uma após 15 anos, devido a trombose do anel e invasão das hastes por fibrose do miocárdio, e outra após 10 anos, devido a fenômenos tromboembólicos de repetição.

O total de próteses retrocadas em posição tricúspide foi em número de 4 , sendo 2 biopróteses de dura-máter, 1 bioprótese tipo Labcor e 1 tipo Starr-Edwards.

Com relação às próteses retrocadas em posição aórtica, de um total de 67 próteses, 51 eram de duramáter, 8 de pericárdio bovino tipo Incor, sendo 6 de alto perfil e 2 de baixo perfil, 3 próteses de Lillehei-Kaster, 1 prótese de Björk-Shiley, 1 prótese de Starr-Edwards, 2 biopróteses de porco tipo Biocor e 1 bioprótese de pericárdio bovino tipo lonescu. Com relação às 51 biopróteses de dura-máter aórticas retiradas, as causas foram: insuficiência por rotura do tecido em 32 casos, calcificação em 12, endocardite infecciosa em 4 e calcificação associada à rotura em 3 . O tempo médio de retroca foi, respectivamente, de 7,$2 ; 7,4 ; 8,3$ e 6,7 anos.

Das 9 próteses de pericárdio bovino, 2 de modelo Incor de alto perfil foram retrocadas por calcificação, com um tempo médio de evolução de 3,5 anos. Duas outras do mesmo modelo foram substituidas, com 2,5 anos de evolução média, por escape paravalvular, 1 por endocardite infecciosa, com 3 anos de evoluçāo e 1 por estenose, com 2 anos de evolução. Das biopróteses de pericárdio bovino de baixo perfil, as 2 foram retrocadas após 1 ano, sendo 1 por rotura e a outra por defeito de confeccção. A bioprótese de pericárdio tipo lonescuShiley foi substituída após 6 anos, por rotura do tecido. Duas próteses tipo Biocor foram retrocadas, 1 com 2 e outra com 6 anos de evoluçāo, por endocardite infecciosa. Com relação às próteses mecânicas, as 3 de Lillehei-Kaster foram substituídas com 3,6 e 9 anos, sendo, respectivamente, devido a trombose, nos 2 primeiros casos, e a tromboembolismo, no último. Um paciente teve sua prótese de Björk-Shiley retrocada, por trombose, após 6 anos de evoluçāo e, em 1 paciente, a prótese de Starr-Edwards foi substituída, devido a abscesso do anel por endocardite infecciosa. Em 4 pacientes aórticos, foi somente trocada a bola da prótese de StarrEdwards, após um tempo médio de 11,3 anos, com variação de 2 a 16 anos.
Os procedimentos cirúrgicos associados à retroca valvular foram realizados em $34(23,4 \%)$ pacientes. A plástica de De Vega na valva tricúspide foi feita em 10 pacientes, 9 associada a retroca valvular mitral e 1 à dupla retroca mitro-aórtica. A troca da valva aórtica foi realizada em 6 pacientes, associada a retroca mitral; 3 pacientes foram submetidos a revascularização do miocárdio, 2 deles associados a retroca aórtica e 1 a retroca mitral. Três comissurotomias mitrais, 4 plásticas de Kay em mitral, 1 ventriculoseptoplastia, 1 fechamento de fistula aórtica-ventrículo direito e 1 ampliaçāo do anel aórtico foram associados à retroca aórtica.

Em 1 paciente submetido a retroca mitro-aórtica, também se realizou a ampliação do anel aórtico; 1 plástica de valva aórtica e 1 troca de valva tricúspide foram associadas à retroca valvular mitral.

Com relação à classe funcional (New York Heart Association - NYHA) no pré-operatório nos 70 pacientes mitrais, $10 \%$ encontravam-se em classe funcional (CF) II (7 pacientes); 31,4\% (22 pacientes) encontravam-se em CF III e $41(58,6 \%)$ em CF IV. Dos 54 pacientes aórticos, $11,1 \%$ (6 pacientes) encontravam-se em CF II, 46,3\% (25 pacientes) em CF III e 42,6\% (23 pacientes) em CF IV. Dos pacientes mitro-aórticos no pré-operatório, num total de 13 pacientes, encontravam-se em CF II $1(7,7 \%)$ paciente, em CF III $4(39,7 \%)$ pacientes e em CF IV $8(61,5 \%)$ pacientes.

Três $(2,06 \%)$ pacientes mitro-tricuspideos encontravam-se em CF IV, pré-operatoriamente, e $1(0,66 \%)$ paciente submetido a retroca valvular tricúspide encontrava-se em CF III.

Dos mitrais, 4 pacientes já haviam sido submetidos a comissurotomia previamente à retroca, sendo, portanto, a terceira operação. Outros 6 pacientes foram submetidos a segunda retroca mitral, sendo que, em 1 paciente, esta era a quarta operaçāo. Dentre os aórticos, 9 pacientes foram submetidos a segunda retroca e 1 a terceira retroca. Um paciente mitro-tricuspídeo foi submetido a terceira dupla retroca. Nos mitro-aórticos, 2 pacientes realizaram a segunda dupla retroca, 1 paciente a terceira retroca mitral e 1 paciente a quarta retroca aórtica.

\section{RESULTADOS}

A mortalidade imediata (30 dias) global foi de $8,3 \%$, correspondente a 12 pacientes. A principal causa de óbito foi o baixo débito cardiaco ocorrendo em 10 pacientes, sendo 7 mitrais, mitro-aórtico e 2 submetidos a retroca mitro-tricuspidea. Os outros 2 pacientes que faleceram no pós-operatório imediato tinham sido submetidos a retroca aórtica, tendo como causa o sangramento difuso por coagulopatia, em 1 caso, e septicemia no outro. 
POMERANTZEFF, P. M. A.; ABREU, M. C. S.; AMATO, M.; MORETTI, M.; AULER JÚNIOR, J. O. C.: GRINBERG, M.; TARASOUTCHI,

F.; MANSUR, A.; DIAS, A. R.; BITTENCOURT, D.; STOLF, N. A. G.; VERGINELLI, G.; JATENE, A. D. - Retroca valvular. Rev. Bras. Cir. Cardiovasc., 2(3): 180-188, 1987.

Dos pacientes mitrais que foram a óbito, 5 encontam-se em CF IV e 2, em CF III.

Os 2 pacientes aórticos que foram a óbito imediato encontravam-se em classe funcional IV. A mortalidade imediata, nos mitrais, foi, portanto, de $10 \%, 7$ pacientes em 70 , e nos aórticos, de $3,7 \%$, ou seja, 2 pacientes em 54.

Nos mitro-aórticos, a mortalidade imediata foi de $7,7 \%$ e, dos 3 pacientes submetidos a dupla retroca mitro-tricuspídea, todos em classe funcional IV no pré-operatório, apenas 1 sobreviveu, tendo os 2 óbitos ocorrido por baixo débito.

As principais complicaçōes ocorridas no período de pós-operatório imediato, considerando-se os óbitos imediatos, foram o baixo débito, as arritmias, o sangramento, além da síndrome pós-pericardiotomia, acidente vascular cerebral e insuficiência renal.

O baixo débito ocorreu em $16(11 \%)$ pacientes, sendo 7 mitrais, 4 aórticos, 3 mitro-aórticos e 2 (11\%) mitrotricuspídeos. Vários tipos de arritmias ocorreram em 15 $(10,3 \%)$ pacientes; sangramento pós-operatório significativo foi constatado em $11(7,5 \%)$ casos, sendo necessária reoperação, para revisão de hemostasia, em 8 $(5,5 \%)$.

Dos 11 pacientes que apresentaram sangramento pós-operatório, 7 eram mitrais, síndrome pós-pericardiotomia foi diagnosticada em $7(4,8 \%)$ pacientes; $6(4,1 \%)$ pacientes apresentaram acidente vascular cerebral isquêmico, $6(4,1 \%)$ insuficiência renal aguda, $5(2,0 \%)$ foram recuperados após parada cardiaca pós-operatória, $4(2,7 \%)$ evoluíram com insuficiência respiratória, 2 $(1,3 \%)$ pacientes foram reoperados por defeito de confecção de prótese, $2(1,3 \%)$ foram reoperados por tamponamento.

Como complicaçōes tardias após a retroca em posição mitral, tivemos 1 caso de anemia por hemólise, com 27 meses de evolução, em bioprótese de pericárdio bovino tipo Incor de alto perfil, sem necessidade de nova troca, 1 caso de sangramento devido a anticoagulação aos 6 meses de evolução, em paciente portador de prótese mecânica tipo Omnicarbon, e 1 caso de substituição por defeito de confecçāo da bioprótese de pericárdio bovino tipo Incor de baixo perfil, aos 18 meses de evolução.

$\mathrm{Na}$ posição aórtica, em relação às complicações tardias, tivemos 1 caso de escape paravalvular em bioprótese de pericárdio bovino tipo Incor de alto perfil, aos 18meses de evolução, 1 caso de insuficiência mitral, que necessitou de tratamento cirúrgico, 12 meses após a retroca aórtica. Nos mitro-aórticos, houve 1 caso de escape paravalvular em bioprótese aórtica de pericárdio bovino tipo Incor alto perfil, aos 12 meses de evoluçāo.

Tivemos apenas 2 óbitos tardios. Um, 20 meses após retroca aórtica, por insuficiência cardiaca conges- tiva, e o outro, no pós-operatório da terceira troca valvular, operação realizada em caráter de emergência, com o paciente levado para a sala de operação sob massagem cardiaca externa e em edema agudo dos pulmões, devido a calcificação da bioprótese de pericárdio bovino tipo Incor de baixo perfil, aos 17 meses de evolução.

No seguimento ambulatorial, encontramos $81,4 \%$ dos pacientes mitrais em CF I e II, com um tempo de acompanhamento de 541 meses/pacientes (Gráfico 3). A curva aturial de sobrevida demonstrou um índice de $85,7 \%$ após 5 semestres (Gráfico 4 ).

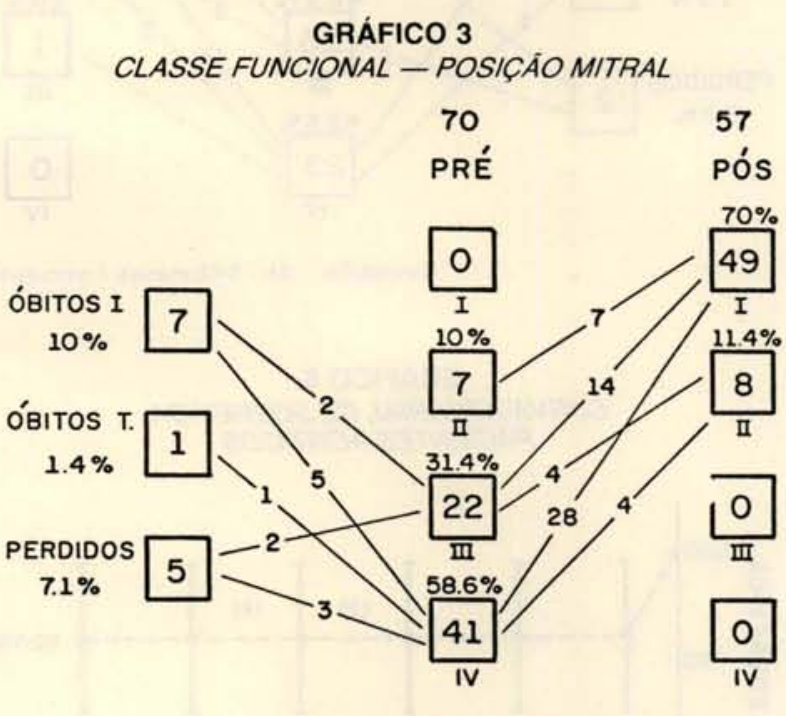

Evoluçôo de 674 mèses / pacientes

GRÁFICO 4

CURVA ATUARIAL DE SOBREVIDA POSICAOO MITRAL

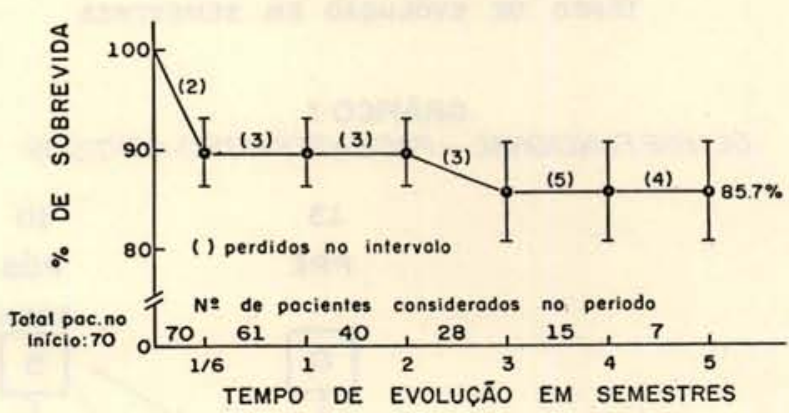

Nos pacientes aórticos, $88,9 \%$ encontravam-se em classe funcional I e II, em um tempo de acompanhamento de 675 meses/pacientes (Gráfico 5). A curva atuarial de sobrevida em 5 semestres mostrou um índice de $91,3 \%$ (Gráfico 6).

Em pacientes submetidos a retroca mitro-aórtica, encontramos $61,5 \%$ em CF I e II, com um tempo de acompanhamento de 155 meses pacientes (Gráfico 7). A curva atuarial de sobrevida demonstrou índice de $92,3 \%$, após 5 semestres (Gráfico 8). 
POMERANTZEFF, P. M. A.; ABREU, M. C. S.; AMATO, M.; MORETTI, M.; AULER JÚNIOR, J. O. C.; GRINBERG, M.; TARASOUTCHI,

F.; MANSUR, A.; DIAS, A. R.; BITTENCOURT, D.; STOLF, N. A. G.; VERGINELLI, G.; JATENE, A. D. - Retroca valvular. Rev. Bras. Cir. Cardiovasc., 2(3): 180-188, 1987

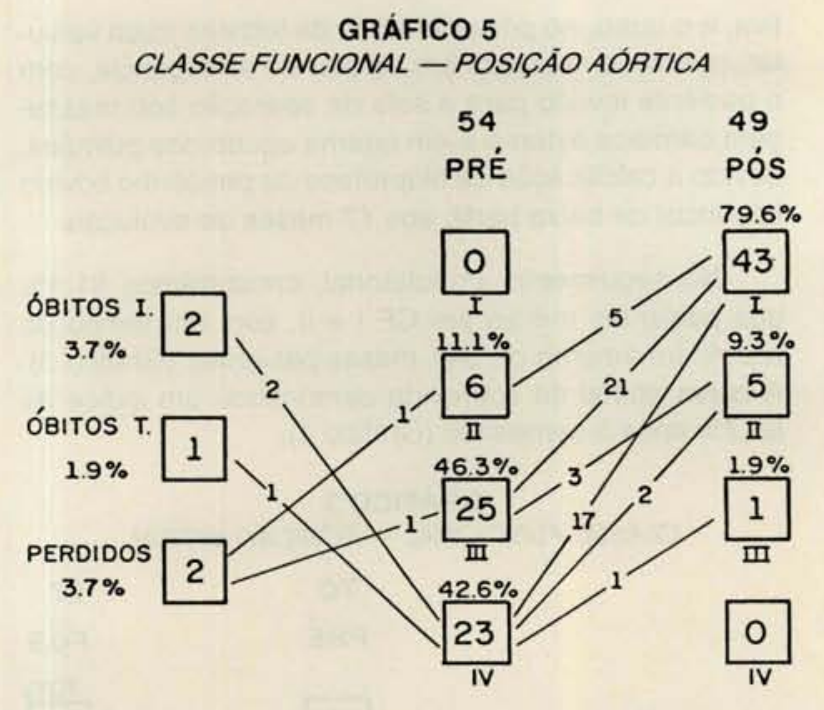

Evolução de 541 meses/pocientes

GRÁFICO 6

CURVA ATUARIAL DE SOBREVIDA PACIENTES AOORTICOS

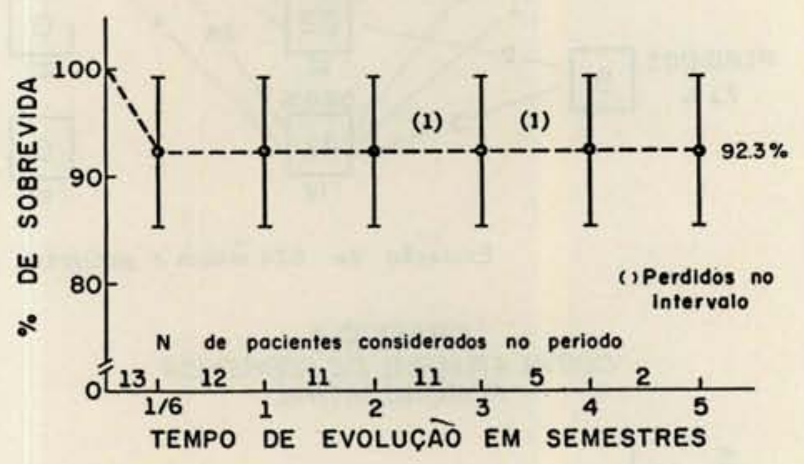

GRÁFICO 7

CLASSE FUNCIONAL - PACIENTES MITRO-AORTICOS

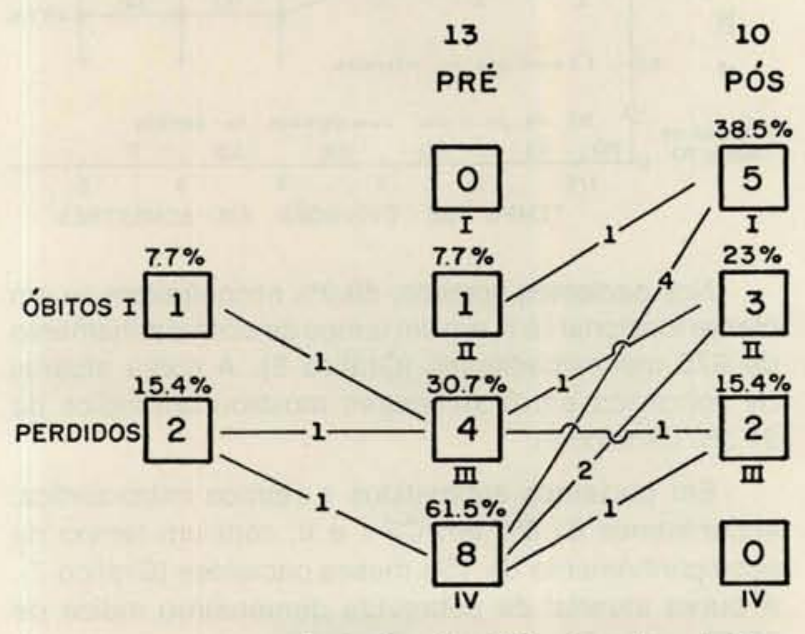

Evoluçāo de 155 m.eses / pacientes
GRÁFICO 8

CURVA ATUARIAL DE SOBREVIDA PACIENTES MITRO-AORTICOS

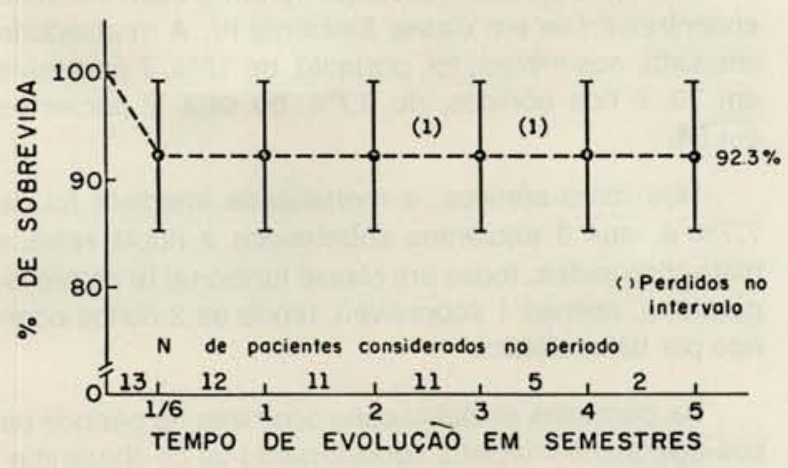

O paciente mitro-tricuspídeo, após seguimento de 30 meses, encontra-se em CF I. Do total de pacientes mitrais, 4 foram submetidos a retroca, sendo, nestes, a terceira operação, sem óbito neste grupo, e 6 pacientes, a segunda retroca, também sem óbito. No grupo dos aórticos, 9 pacientes foram submetidos a segunda retroca, com 1 óbito.

\section{DISCUSSĀO}

Em nossa casuística, houve um número maior de reoperaçōes em posição mitral $(48,2 \%)$ havendo predominância do sexo feminino.

BOSH et alii ${ }^{2}$, analisando 89 pacientes submetidos a reoperação, sendo 64 próteses mecânicas e 25 biopróteses, também encontraram uma porcentagem maior de reoperaçōes mitrais, em número de 46 , correspondendo a $5 \%$. SYRACUSE \& MALM ${ }^{18}$, analisando 89 próteses reoperadas, todas mecânicas, relatam o dobro de reoperações de próteses aórticas em relação à mitral, tendo, também, encontrado um predomínio do sexo feminino, em posição mitral.

Como temos utilizado, predominantemente, biopróteses, isto explica o fato de que as principais causas de retroca valvular sejam a rotura e a calcificação do tecido. O nosso número de reoperaçōes devido a escape paravalvular ( 3 em posição aórtica e 1 em posição mitral) pode ser considerado baixo, se comparado a outros autores. SANDZA Jr. et alii ${ }^{16}$, em uma série de 75 pacientes submetidos a reoperaçōes valvulares, encontraram 22 pacientes com escape paravalvular. $\mathrm{COHN}$ et alii ${ }^{4}$ relatam 58 reoperaçōes, representando $4,5 \%$ de um total de 1282 operações consideradas como primeira intevenção, com percentagens iguais para próeses mecânicas e biológicas e onde a causa da reoperação foi, em $25 \%$ dos casos, deiscência da válvula.

Em uma série de 858 pacientes aórticos e $801 \mathrm{mi}$ trais com mais de 15 anos de idade, submetidos a troca 
POMERANTZEFF, P. M. A.; ABREU, M. C. S.; AMATO, M.; MORETTI, M.; AULER JUNIOR, J. O. C.; GRINBERG, M.; TARASOUTCHI F.: MANSUR, A.; DIAS, A. R.; BITTENCOURT, D.: STOLF, N. A. G.; VERGINELLI, G.; JATENE, A. D. - Retroca valvular Rev. Bras. Cir. Cardiovasc., 2(3): 180-188, 1987

valvar por bioprótese de Hancock, com tempo de acompanhamento de 7 anos, OYER et alii ${ }^{12}$ relatam 52 reoperações, sendo 28 aórticos e 24 mitrais. As causas de reoperaçōes, segundo estes autores, foram: endocardite bacteriana em 15 pacientes aórticos e 6 mitrais, e falência primária do tecido em 4 pacientes aórticos e 10 mitrais. Como se pode observar ${ }^{12}, 54 \%$ dos pacientes que necessitaram retroca aórtica e $25 \%$ dos que necessitaram retroca mitral tiveram, como causa de reoperação, a endocardite bacteriana, o que difere da nossa casuística, onde temos $9 \%$ de reoperaçōes de biopróteses por endocardite em posiçāo mitral e 10,3\% em posição aórtica.

KLÖVEKORN et alii ${ }^{8}$ relatam 1915 trocas valvares, de abril de 1974 a fevereiro de 1982, em 1602 pacientes sendo 874 tipo Björk-Shiley, 1041 biopróteses porcinas (de Hancock e de Carpentier-Edwards), sendo que 25 pacientes foram reoperados devido a falência de 31 próteses. As causas de reoperação foram endocardite em 14 pacientes, com tempo médio de evolução de 26 meses, degeneraçāo do tecido em 8 pacientes, após 30 meses de evolução média, e 3 pacientes foram reoperados por escape paravalvular, após 9 meses de evolução média. Relatam, ainda, que a maior inicidência de endocardite foi encontrada em pacientes com idades entre 40 e 50 anos e que a causa das reoperações em pacientes jovens estava relacionada a degeneração tecidual e calcificação. Em nossos casos, a maior causa de reoperaçōes em pacientes jovens foi, também, a calcificaçāo, sendo que os dados quanto à endocardite diferem daqueles dos autores. Segundo os mesmos, a endocardite e o escape paravalvular aparecem nos dois primeiros anos de evolução, ao contrário do nosso estudo, onde as biopróteses reoperadas por endocardite tinham tempo médio de evolução de 6 anos em posição mitral e 7 anos em posição aórtica, acrescido da ressalva de que não estamos considerando todos os pacientes submetidos a troca valvar por bioprótese e sim analisando um grupo de pacientes operados no período entre janeiro de 1984 e junho de 1986.

VON DER EMDE et alii ${ }^{20}$ relatam, em trabalho sobre retroca valvular, que os casos de trombose mecânica estavam associados a interrupção abrupta do programa de anticoagulação. OLESEN et alii ${ }^{11}$, em trabalho sobre 262 pacientes nos quais utilizaram próteses mecânicas de Lillehei-Kaster, em acompanhamento de 5 anos, reoperaram 8 pacientes, sendo 4 por escape paravalvular, 3 por endocardite bacteriana e 1 por trombose de válvula, sendo que todos os pacientes utilizavam anticoagulantes orais.

BRUIN et alii ${ }^{3}$ estudaram 847 pacientes submetidos a trocas valvares por próteses mecânicas, sendo que 38 destes foram reoperados. A causa mais freqüente de reoperação foi endocardite bacteriana em 15 pacientes e escape paravalvular em 7 , sendo que 2 pacientes foram reoperados, devido a trombose da prótese.
Em nossos pacientes, a causa mais freqüente de reoperação, nas próteses mecânicas, foi trombose.

Com relação à técnica operatória, temos utilizado serras circulares de oscilação, para abertura ou reabertura do esterno, com tração do mesmo, prevenindo-se, desta forma, laceração do miocárdio; realizamos a dissecção procurando o plano cirúrgico adequado e, quando possivel, a dissecção é feita com bisturi elétrico, para melhor hemostasia. Temos realizado a abertura de pleura esquerda nos casos de maior aderência, diminuindo, desta forma, a área de descolamento, evitando sangramento pós-operatório. Com a pleura esquerda aberta, não existe dificuldade para o acesso ao anel mitral e tampouco para as manobras de retirada do ar do coração. Na maioria das vezes, a perfusão foi feita pela aorta, sendo usado o oxigenador de bolhas descartável.

A cardioplegia foi utilizada na maioria dos casos, sendo que, em alguns mitrais, foi utilizada a perfusão coronária contínua com a aorta pinçada, perfusāo esta realizada a partir de dispositivo (perfursor) conexionado à linha arterial.

Para implante de nova prótese, utilizamos pontos passados no anel em $U$ ou em $X$, quando em $U$ ancorados com Teflon, nos casos de anéis frágeis. Provavelmente, o fato de sempre termos utilizado pontos separados para implante de próteses explique a baixa incidência de reoperações por escape paravalvular.

Temos implantado, perferentemente, nas reoperações, biopróteses, justificando o seu uso pelos bons resultados apresentados pelas mesmas e pela dificuldade de controle da anticoagulação, em nosso meio.

RODEWALD et alii ${ }^{15}$, em evisāo da literatura, mostram que a mortalidade operatória nas retrocas valvulares tem variado entre $1,8 \%$ e $31 \%$ e que $17 \%$ dos óbitos imediatos são devidos a acidentes de técnica operatória.

ENRIGHT et alii ${ }^{7}$ relatam $12,5 \%$ de mortalidade hospitalar em retrocas mitrais, chamando a atençāo para o fato de que a mortalidade está relacionada com o estado clínico do paciente.

MAGILLIGAN Jr. et alii ${ }^{9}$, em estudo de 12 anos de acompanhamento de 951 biopróteses de porco, em 817 pacientes, relatam reoperação em 56 pacientes, com mortalidade hospitalar de $12,5 \%$, sendo as principais causas de óbito o sangramento em 2 pacientes e falência miocárdica em outros dois.

PARR et alii ${ }^{13}$ relatam mortalidade de $42,4 \%$, em reintervençōes de urgência.

WIDEMAN et alii ${ }^{21}$, em 200 retrocas valvulares aórticas, apresentam mortalidade imdiata de $5 \%$ correspondente a 10 pacientes, sendo que 6 faleceram por falência cardiaca, 2 por hemorragia devido a acidente 
de esternotomia, e 2 por problemas neurológicos. Destes pacientes, 7 óbitos ocorreram na primeira retroca valvular e 3 óbitos na segunda, ou terceira operaçăo. Consideram, como fatores que aumentam o risco cirúrgico, a classe funcional IV, a endocardite da prótese, o tempo de longo e pinçamento aórtico e a parada anóxica isquêmica, mesmo com resfriamento.

Em nosso meio, DONELLI et alii ${ }^{6}$, analisando 64 pacientes submetidos a reoperaão valvular, encontraram mortalidade global de $17 \%$, sendo $18 \%$ para reoperaçōes em bioprótese e $15 \%$ para próteses mecânicas com mortalidade imediata para a mitral de $23 \%$ e aórtica de $8 \%$.

A nossa mortalidade imediata também foi menor em aórticos do que em mitrais, respectivamente 3,7\% e $10 \%$, em concordância com a literatura; em pacientes mitro-aórticos, a mortalidade foi de $7,7 \%$. No grupo de segunda retroca mitral, não tivemos óbitos e, no grupo de segunda retroca aórtica, tivemos 1 óbito; portanto, a mortalidade não aumento com o número de operaçōes prévias.

Em nossa casuística, a principal causa de óbito foi o baixo débito cardico, sendo que, dos 7 mitrais que faleceram no pós-operatório imediato, 4 tinham calcificação da prótese, com estenose severa e em classe funcional IV.

Consideramos, com conclusão, ser de grande importância, para reduzir a mortalidade operatória, reoperar os pacientes antes da severa falência hemodinâmica, um planejamento cirúrgico adequado pela técnica já descrita, além de monitorização da coagulaçāo sangüínea, evitando, assim, sangramento pós-operatório, e uma grande atençāo do cirurgiāo para a proteçāo miocárdica.

RBCCV 44205-39

POMERANTZEFF, P. M. A.; ABREU, M. C. S.; AMATO, M.; MORETTI, M.; AULER JÚNIOR, J. O. C. GRINBERG. M.; TARASOUTCHI, F.; MANSUR, A.; DIAS, A. R.; BITTENCOURT, D.; STOLF, N. A. G.; VERGINELLI, G.; JATENE, A. D. - Replacement of heart valves prostheses. Rev. Bras. Cir. Cardiovasc., 2(3): 180-188, 1987.

ABSTRACT: Replacement of valvular prosthesis is an increasingly frequent procedure in heart surgery. Better results are attained with the observation of correct indication and improved surgical technique. In the period of January 1984 to June 1986,145 patients were submitted to prosthesis replacement, at our Institution. These patients received a total of 157 prostheses and 4 had their Starr-Edwards valve ball replaced due to ball variance. Six patients were submitted to a third valvular replacement in the mitral position, witiouth deaths. Nine patients had a third valvular replacement in the aortic position with 1 death in the immediate postoperative period. Rupture or calcification of the dura mater leaflets were the main reason for the indication of prosthetic replacement. In the mitral position 41 patients presented rupture of the leaflets and 19 showed calcification. In the aortic position 32 bioprostheses underwent rupture and 12 , calcification. Bio-prostheses were utilized in the majority of cases of replace the dysfunctioning prostheses. Porcine bioprostheses were implanted in 63 cases and bovine pericardial bioprostheses, in 35 . Immediate mortality was $8.3 \%$ (12 patients); low cardiac output was the main cause of death. The most frequent immediate complications were low cardiac output, arrhythmias and bleeding. In the preoperative period $90 \%$ of the patients were in functional classes III and IV (NYHA). After the replacement, $89 \%$ of the aortics and $82 \%$ of the mitral were in functional classes I an II.

DESCRIPTORS: heart valves, prostheses, replacement.

\section{REFERÊNCIAS BIBLIOGRÁFICAS}

1 ANDERSON, R. P.; BONCHEK, L. I.; GRUNKEMIER, G. L.; LAMBERT, L. E.; STARR, A. - The analysis and presentation of surgical results by actuarial methods. J. Surg., Res. 16(3): 224-230, 1974.

2 BOSH, X.; POMAR, J. L.; PELLETIER, L. C. - Early and late prognosis after reoperation for prosthetic valve replacement. J. Thorac. Cardiovasc. Surg., 88(4): 567-572, 1984.

3 BRUIN, M. J.; LACQUET, L. K.; SKOTNICKI, S. H.; VICENT, J. G.; VAN DER MEEER, J. J. - Reoperation for prosthetic heart valve replacement. Thorac. Cardiovasc. Surg., 34(1): 12-16, 1986.
4 COHN, L. H.; KOSTER, J. K.; VANDE-VANTER, S.; COLLINS, J. J. - The in-hispital risk of replacement of dysfunctional mitral and aortic valves. Circulation, 66(1): 153-156, 1982.

5 COPELAND, J. C.; GRIEPP, R. B.; STINSON, E. B.; SHUMWAY, N. E. - Long-term follow-up after isolated aortic valve replacement. J. Thorac. Carviovasc. Surg., 74(6): 875-885, 1977.

6 DONELLI, L. A. C.; MATSUMOTO, L. F; GANDRA, S. RIVETTI, L. A.; FERLANTI, L. E. S.; SANTOS, R. G.; FELIPOZZI, H. J. - Reoperaração em portadores de próteses valvulares cardiacas: análise de 64 casos. Arq. Bras. Cardiol., 45(6): 407-411, 1985. 
POMERANTZEFF, P. M. A.; ABREU, M. C. S.; AMATO, M.; MORETTI, M.; AULER JÚNIOR. J. O. C.; GRINBERG, M.; TARASOUTCHI, F.; MANSUR, A.; DIAS, A. R.; BITTENCOURT, D.; STOLF, N. A. G.; VERGINELLI, G.; JATENE, A. D. - Retroca valvular Rev. Bras. Cir. Cardiovasc., 2(3): 180-188, 1987.

7 ENRIGHT, L. P.; MITCHELL, R. L.; WILBUR, B. G.; SAAH, I. D.; CONSTANTINO, R. T.; KLUCHAUPT, M. - Longterm experience with mitral valve replacement: an evolving strategy for prosthesis selection. Am. Heart J., 109(6): 1360-1368, 1985.

8 KLÖVEKORN, W. P.; STRUCK, E.; HOLPER, K.; MEISNER, H.; SEBENING, F. - Causes of valve failure and indications for reoperation after bioprosthetic cardiac valve replacement. In: COHN, L. H. \& GALLUCCI, V. eds. Cardiac boprostheses. New York, Yorke Medical Books, 1982, p. 530-538.

9 MAGILLIGAN Jr., D. J.; LEWIS, J. W.; TILLEY, B.; PETERSON, E. - The porcine bioprosthetic valve: twelve years later. J. Thorac Cardiovasc. Surg/. 89(4): 499-507, 1985.

10 ODELL, J. A. - Calcification on porcine bioprosthesis in children. In: COHN, L. H. \& GALLUCCI, V. eds. Cardiac bioprosthesis. New York, Yorke Medical Books, 1982. p. 231-237.

11 OLESEN, K. H.; RYGG, I. H.; WENNEVOLD, A.; NYBOE, J. - Long-term follow-up in 262 patients after aortic valve replacement with the Lillehei-Kaster prosthesis: overall results and prosthesis related complications. Eur. Heart J., 7(9): 808-816, 1986.

OYER, P. E.; STINSON, E. B.; MILLER, D. C.; JAMIESON, S. W.; REITZ, B. A.; BAUMGARTNER, W.; SHUMWAY, N. E. - Clinical analysis of the Hancock porcine bioprosthesis. In: COHN, L. H. \& GALLUCCI, V. eds. Cardiac bioprostheses. New York, Yorke Medical Books, 1982. p. 539-551.

13 PARR, G. V.; KIRKLIN, J. W.; BLACKSTONE, E. H. The early risk of re-replacement of aortic valves. Ann. Thorac. Surg., 23(4): 319-322, 1979.

14 PUIG, L. B.; VERGINELLI, G.; BELLOTTI, G.; KAWABE, L.; FRACK, C. C. R.; PILEGGI, F.; DÉCOURT, L. V.; ZERBINI, E. J. - Homologous dura-mater cardiac valves: preliminary study of 30 cases. J. Thorac. Cardiovasc. Surg., 64(1): 154-160, 1972.

15 RODEWALD, G. J.; GUNTAN, J.; BANTEA, C.; KASMAR, P.; KREBBER, E. J.; RODIGER, W.; TILSNER, V. The risk of reoperation in acquired valvular heart disease. Thorac. Cardiovasc. Surg., 28(1): 77-88, 1980.

16 SANDZA Jr., J. G.; CLARK, R. E.; FERGUSON, R. B.; CONNORS, J. P.; WELDON, C. S. - Replacement of prosthetic heart valves: a fifteen-year experience. J. Thorac. Cardiovasc. Surg., 74(6): 864-874, 1977.

17 STARR, A. - Starr-Edwards ball valve. In: LEFRAK, E. A. \& STARR, A. Cardiac valve prosthesis. New York, Appleton-Century-Crofts, 1979. p. 67-117.

18 SYRACUSE, D. L. \& MALM, J. R. - Prosthetic valve reoperations factor influency early and survival. J. Thorac. Cardiovasc. Surg., 77(3): 346-353, 1979.
19 TEPLEY, J. F.; GRANKEMEIER, G. L.; SUTHERLAND, H. D. A.; LAMBERT, I.; JOHNSON, V.; STARR, A. - The ultimate programs after valve replacement: an assessement at twenty years. Ann. Thorac. Surg. 32(2): 111-119, 1981

20 VON DER EMDE, J.; KOCKERLING, F.; REIN, J.; HERTLEIN, A. - Measures of preservation and technical problems during reoperations in cardiac surgery. Thorac. Cardiovasc. Surg., 34(1): 5-11, 1986.

21 WIDEMAN, F. E.; BLASCKSTONE, E. H.; KIRLIN, J. W.; KARP, R. B.; KOUCHOUKOS, N. T.; PACIFICO, A. D. - Hospital mortality of re-replacement of the aortic valve: incremental risk factors. J. Thorac. Cardiovasc. Surg., 82(5): 692-698, 1981.

\section{Discussão}

\section{DR. B. GIBSON F. COSTA \\ Salvador, $B A$}

As reoperaçōes das próteses valvulares têm sido uma constante, nos Serviços de cirurgia cardiaca, assim como um desafio à tecnologia, a clínicos e cirurgiōes. Quando nos propomos a implantar uma prótese, já sabemos que, mais cedo ou mais tarde, essa mesma prótese será substituída, frente às lesões apresentadas. Sabemos de longa data, que foi possivel substituir as valvas cardiacas naturais em disfunçāo, por válvulas cardiacas artificiais mecânicas e biológicas. A substituição valvar se consagrou entre os cardiologistas como eficiente processo terapêutico cirúrgico e, em si, constitui cerca de 1/3 das casuísticas, só perdendo para os processos de revascularização do miocárdio. Os resultados são sempre favoráveis, uma vez que melhora as condiçōes hemodinâmicas e prolonga a vida do paciente, principalmente nos casos com boa contratilidade ventricular. Falar de próteses valvulares seria falar de um complexo problema e, apesar do avanço dentro do campo da tecnologia, com respeito à feitura da prótese, a qualidade do material que compõe a prótese, a durabilidade, o funcionamento, assim como os meios de conservaçāo, estes substitutos ainda constituem um assunto polêmico, onde se sobressaem a resistência, o desempenho funcional e as inúmeras complicaçōes, que levam os enfermos às substituiçōes (trocas e retrocas). Nenhuma prótese confeccionada até a presente data tem-se mostrado livre, seja a curto ou a longo prazo, de uma série de complicações, tais como: a degeneração tecidual, a trombose, a fibrose, a proliferação do tecido endotelial, a retraçāo-rigidez do tecido e a calcificaçāo, que se associam entre si, modificando a dinâmica da unidade móvel da prótese (bola, disco ou cúspides). Todas as próteses, tanto mecânicas como biológicas, atuam dentro de um mesmo princípio hemodinâmico e nenhuma foge à regra de ser substituída dentro de um período de tempo que, excepcionalmente, ultrapassa a 10 ou 12 anos. Em prin- 
POMERANTZEFF, P. M. A.; ABREU, M. C. S.; AMATO, M.; MORETTI, M.; AULER JÚNIOR, J. O. C.; GRINBERG, M.; TARASOUTCHI, F.; MANSUR, A.; DIAS, A. R.; BITTENCOURT, D.; STOLF, N. A. G.; VERGINELLI, G.; JATENE, A. D. - Retroca valvular. Rev. Bras. Cir. Cardiovasc., 2(3): 180-188, 1987.

cípio, todas as próteses empregadas são rígidas, mesmo aquelas que têm como base de sustentação (suporte) um simples anel, como, por exemplo, a prótese de Hancock, de flexibilidade muito reduzida que, em sua função, não corresponde à dinâmica orificial natural, que, por sua vez, é dependente da ação da própria musculatura miocárdica paravalvar, que, assim, rege o mecanismo de abertura e fechamento do aparelho valvar. Todas as próteses utilizadas, nos dias atuais, são parcialmente inertes, tendo apenas, como elemento móvel, a bola, o disco e as cúspides, ficando os demais componentes em stado de franca imobilidade (suporte de sustentação). Toda esta exposição, que afirma condiçōs já conhecidas com relação às próteses, tão cedo não se modificará, até que tenhamos uma prótese dinâmica em seu todo, que, possivelmente, fará desaparecer todas as complicações que têm levado os enfermos às trocas e retrocas. No presente trabalho, observamos que, em posição mitral, 6 enfermos foram submetidos a terceira troca valvular, sem óbito; em posição aórtica, 9 enfermos foram, também, submetidos a terceira troca valvular, apenas com 1 óbito. Esta afirmação vem provar, mais uma vez, que carecemos de uma prótese ideal e que nos ofereça alta confiabilidade em sua duração e funcionamento. Seria, então, aquele prótese que se mantivesse dinâmica e jamais contraísse complicaçōes. Frente a estes problemas, quero crer que os autores farão a quarta troca valvular e as lesōes a serem encontadas serāo idênticas àquelas que foram observadas quando das trocas anteriores. Atualmente, não há por onde fugir a esta determinação, mesmo que se tenha o máximo de cuidado na escolha da prótese e nas instruções no preparo da mesma, ao ser implantada. As complicações às quais se referem são relativamente freqüentes, nesses tipos de cirurgia. A curva atuarial aqui apresentada, em enfermos aórticos e mitrais, respectivamente, $89 \%$ e $82 \%$, está dentro dos padrões aceitáveis e não poderia ser melhor. Quem quer que seja, ao elaborar um trabalho dentro deste contexto, provavelmente dirá o mesmo e que se encontra inserido no presente trabalho. $O$ meu ponto de vista é fazer, aqui, um comentário simples e construtivo e, em hipótese alguma, trazer um trabalho em paralelo, a fim de mostrar experiência, que, por certo, demonstraria dados praticamente idênticos a esses aqui apresentados. O enfermo e o cirurgião pagam um grande tributo por terem que usar válvulas cardiacas artificiais parcialmente dinâmicas. O presente trabalho é muito bom e sério, uma vez que nele nada existe que permita uma análise crítica contundente. A segurança deste trabalho, dentro do campo da técnica operatória, da proteção miocárdica e dos cuidados de um pós-operatório bem orientado, diz bem de melhores condições aos enfermos e maior sobrevida. Meus cumprimentos aos autores.

\section{DR. MÁRIO DE ABREU (Encerrando)}

Agradecemos as palavras do Dr. Gibson e concordamos que o número de reoperações de próteses valvulares, atualmente, é grande, devido aos vários problemas existentes com as mesmas. Sem dúvida, nos próximos anos, teremos que nos defrontar muitas vezes com esta situação e acreditamos que o aprimoramento da técnica operatória, da proteção miocárdica e dos cuidados pósoperatórios certamente permitirão a obtenção e resultados cada vez melhores. 